\title{
Preliminary Report on the Flora of Southern Hidaka, Hokkaido (Yezo). XXIV.
}

\author{
By
}

\section{Hiroshi Hara.}

Received November $2,193 \%$.

\section{Compositae. ${ }^{1)}$}

Achillea L.

$563^{(1) * A c h i l l e a ~ p u l c h r a ~ K o I d z u m ~ i n ~ B o t . ~ M a g . ~ T o k y o ~ X X X I I, ~ p . ~} 59$ (1918).

Syn. Achillea speciosa var. pulchra NaKaI, Veg. Apoi p. 14 \& 70 (1930).

Nom. Jap. Akabana-yezzonokogirisô (KoIDz. 1918).

Hab. in grassy places in Mt. Apoi, etc. Fl. mid Jul.-Aug.

f. alba Hara, f. nov.

Ligula floris alba.

Typus. Yezo: prov. Kushiro: Kiritap (H. HARA-Aug. 8, 1936).

Hab. along Horoman R., between Shoya and Saruru.

Endemic species in Yezo.

"var. angustifolia Hara, var. nov.

Syn. Achillea Ptarmica (non L.) NaKaI, Veg. Apoi p. 14 \& 70 (1930).

Caulis $15-40 \mathrm{~cm}$. altus gracilescens. Folia anguste linearia $2-6 \mathrm{~cm}$. longa $2-4 \mathrm{~mm}$. lata margine serrata vel inciso-serrata. Caput minus. Ligula floris alba.

Nom. Jap. Horoman-nokogirisô (nov.)

Hab. at the mouth of Horomon R. (H. HARA-Sep. 6, 1933-typus). Fl. Aug.--Sep.

**f. rosea HarA, f. nov.

Ligula floris rosea. Cetera ut in var. angustifolio.

Hab. at the mouth of Horoman R. (rare).

This variety is known only in the above locality.

Adenocaulon Hooker.

564 (2) * Adenocaulon adhaerescens Maximowicz, Prim. Fl. Amur. p. 152 (1859).-Komarov, Fl. Mansh. III, p. 620 (1907).-NaKaI, Veg. Apoi p. 70 (1930).

1) Some eritical species of Compositae were collaborated with Mr. Siro Kitamura of Kyoto Imperial University. 
Syn. Adenocaulon bicolor Hooker var. adhaerescens MAKINo in Bot. Mag. Tokyo XIII, p. [240] (1899); XXIII, p. 17.(1909).-Matssumura, Ind. Pl. Jap. II-2, p. 620 (1912).

Nom Jap. Nobuki.

Hab. at the foot of Mt. Apoi, ete. Fl. Aug.

Dist. Kyushu, Shikoku, Honshu, Yezo, Korea, China, Manchuria, Ussuri and Amur.

Anaphalis DC.

$565^{(3) * A n a p h a l i s ~ a l p i c o l a ~ M A K I N o ~ i n ~ B o t . ~ M a g . ~ T o k y o ~ X V I I, ~ p . ~} 151$ (1903).-Matsumura, Ind. Pl. Jap. II-2, p. 621 (1912).-Tatew., Veg. $\Lambda$ poi p. 113 (1928).

*f. robusta Hara, f. nov.

Syn. Anaphalis alpicola Makino, l.c. (1903) quoad pl. ex Samanı.

Anaphails apoiensis NAKAI, Veg. Apoi p. $30 \& 70$ (1930) nom. seminud.

Planta robustior quam typo. Caulis usque ad $40 \mathrm{~cm}$. altus. Folia basilaria ad $2 \mathrm{~cm}$. lata. Flores semper albi.

Nom. Jap. Apoi-hahakogusa (NAKAI 1930).

Hab. in the middle \& upper part of Mt. Apoi and Mt. Horoman. Fl. Jul.-Aug.

Dist. sp. Honshu and Yezo.

$566^{(4) * A n a p h a l i s ~ m a r g a r i t a c e a ~ B e n t h a m ~ e t ~ H o o k e r ~ f i l ., ~ G e n . ~ P l . ~ I T, ~ p . ~}$ 303 (1873).

Syn. Gnaphalium margaritaceum Linnatus, Sp. Pl. ed. 1, II, p. 850 (1753). subsp. angustior (Mrquel) Kitamura et Hara, comb. nov.

*var. angustior Nars In Bot. Mag. Tokyo XL, p. 148 (1926).

Syn. Antennaria cinnamomea DC. var. ? $\beta$. angustior MIques in Ann. Mus. Bot. Lugd.-Bat. II, p. 178 (1866).

Gnaphalium margaritaceum $\beta$. intermedium s. kamtschaticum HERDER in Bull. Soc. Imp. Nat. Moseou XL-1, p. 415 (1867).

?Anaphalis margaritacea var. occidentalis Greene, Fl. Franciscana IV, p. 399 (1897).-FERNALD in Rhodora XIII, p. 27 (1911).Hultén, Fl. Kamt. IV, p. 164 (1930).

Anaphalis margaritacea (non Bentir. et Hooker f.) Matsumura, Ind. Pl. Jap. II-2, p. 621 (1912).-Kudo, Fl. Param. p. 165 (1922) (cum f. latifolia KUdo).--Tatew., Veg. $\Lambda$ poi p. 113 (1928).-Komarov, Fl. Penin. Kamt. III, p. 132 (1930).-TAtewaKi et Kolzayashi, Contr. FI. Aleutian Is. p. 84 (1934).

Anaphalis margaritacea var. cinnamomea NAKAI, Veg. Apoi p. 23 \& 70 (1930) excl. syn. 
Nom. Jap. Yama-hahako.

Hab. in Mt. Apoi, Shoya, Mt. Rakko, etc. Fl. Aug.-Sep.

Dist. var. Honshu, Yezo, Kuriles, Saghalien, Ussuri?, Ochotsk, Kamtehatka and N. America.

Leaves of the specimens in this district are $8-18 \mathrm{~mm}$. wide.

*f. ramosa (NakaI) Hara, comb. nov.

Syn. Anaphalis margaritacea var. ramosa NAKAI, Veg. Apoi p. $23 \& 70$ (1930).

Caulis supra medium dense ramosus.

Nom. Jap. Edauchi-yamahahako (NaKAI 1930).

Hab. at the foot of Mt. Apoi.

var. intercedens HARA, var. nov.

Syn. Anaphalis yedoensis (non Maxim.) T'AKeda in Bot. Mag. Tokyo XXIV, p. 315 (1910).

Caulis simplex erectus $50-80 \mathrm{~cm}$. altus albo-lanatus saepe ab axibus foliorum superiorum ramos tenuis emittens. Folia lineari-lanceolata 5-11 cm. longa 2-7 mm. lata supra incano-lanata subtus dense albo-lanata. Caput ut in var. angustiore.

Nom. Jap. Yezo-kawarahahako (nov.).

Hab. on gravelly river-beds of Saruru R. (H. HARA-Aug. 30, 1933 -type). Fl. late $\Lambda$ ug.-Sep.

Dist. var. Honshu and Yezo.

This is an intermediate form between $\Lambda$. margaritacea subsp. angustior and subsp. yedoensis and is not rare in gravelly places of Ye\%o.

Arnica L.

$567^{(\breve{a})}$ Arnica unalaschcensis Lessing in Linnaea VI, p. 238 (1831).Matsumura, Ind. Pl. Jap. II-2, p. 622 (1912).-Kudo, Fl. Param. p. 170 (1922).-Hultín, Fl. Kamt. IV, p. 193 (1930).-T'Tatewaki et Koßayashi, Contr. Fl. Aleut. Is. p. 85 (1934).

Nom. Jap. Yezo-usagigiku (nov.).

Hab. on Mt. Rakko and Tokachi. Fl. Jul.

Dist. sp. Mid. \& N. Honshu, Yezo, Kuriles, S. Kamtchatka and Aleutian.

Specimens of the district as well as those of Unalaska have nearly glabrous corolla-tubes. The plant of Honshu,, ${ }^{1}$ however, has generally

1) Arnica unalaschconsis Lessing.

var. Tschonoskyi (ILJin) Kitanura et H.arA, comb. nov.

Syn. Arnica Tschonoskyi It.JIn in Trav. Mus. Bot. Acad. Sei. URSS. XIX, p. 119 (1926).

Nom. Jap. Usagi-giku.

Hab. Honshu media et septentrionalis. 
hairy corollat-tubes, and has often less hairy stems, leaves and involucral bracts, although intermediate forms are found.

\section{Artemisia L.}

$568^{(6)}$ Artemisia arctica Lessing in Linnaea VI, p. 213 (1831).-Hultén, Fl. Kamt. IV, p. 176 (1930).-Komarov, Pl. Penin. Kamt. III, p. 150 (1930). -Tatewaki et Kobayashi, Contr. Fl. Aleut. Is. p. 85 (1934).-KItamura in Acta Phytotax. et Geobot. V, p. 88 \& 96 (1936).

Syn. Artemisia norvegica (non Fries) Matsumura, Ind. PI. Jap. II-2, p. 624 (1912).-PAMPanini in Nuovo Giorn. Bot. Ital. n.s. XXXIV, p. 684 (1927).

Nom. Jap. Samani-yomogi.

Hab. on rocks of C. Enrum and Mt. Toyoni (ex Tsushima).

Dist. N. Honshu, Yezo, Kuriles, Saghalien, Kamtchatka, Siberia and N. America.

$569^{(7) * A r t e m i s i a ~ g i g a n t e a ~ K i t a m u r a ~ i n ~ A c t a ~ P h y t o t a x . ~ e t ~ G e o b o t . ~ I I, ~ p . ~}$ 172 (1933); III, p. $91 \& 99$ (1936).

Syn. Artemisia vulgaris L. var. vulgatissima (non BEsser) auct. Jap.

Artemisia vulgaris var. yezoana Kudo, Medic. Pl. Hokkaido no. 97 (1922) cum fig.

Artemisia montana (non Schlechtendau 1846) Pampanini in Nuovo Giorn. Bot. Ital. n.s. XXXVI, p. 461 (1930).

"f. montana (NAKAI) HARA, comb. nov.

Syn. Artemisia vulgaris var. indica f. montana NAKAI in Bot. Mag. Tokyo XXVI, p. 104. (1912).

Artemisia vulgaris var. kamtschatica (non LedeBour) auct. Jap.Tatew., Veg. Apoi p. 114 (1928).

Artemisia coarctata (non Fories) NAKAI, Veg. Apoi p. 70 (1930).

Nom. Jap. Yezo-yomogi.

Hab. at the foot of Mt. Apoi.

"f. electa (Pampanini) Hara, comb. nov.

Syn. Artemisia vulgaris var. latiloba (non Ledeßour) NAKAI, l.c. p. 103 (1912).

Artemisia montana var. a. latiloba Pampanini, l.c. (1930).

Artemisia nipponica Pampanini var. electa Pampanini, 1.c. p. 464 (1930).

Artemisia coarctata var. latiloba NAKaI, Veg. Apoi p. 71 (1930) excl. Syn. INEDEB.

Nom. Jap. $\hat{O}$-yomogi, Yama-yomogi.

Hab. at the foot of Mt. Apoi, Saruru, etc. Fl. Sep. 
* whikotanensis (KITAMURA) HARA, comb. nov.

Syn. Artemisia shikotanensis Krtamura in Aeta Phytotax. et Geobot. III, p. 128 (1934) (ut shikotaensis).

Nom. Jap. Shikotan-yomogi (KItamura 1934).

Hab. Porosanushibetsu.

Dist. sp. IIonshu, Yezo, Kuriles and Saghalien.

$570^{(8)}$ *Artemisia japonica Thunberg, Fl. Jap. p. 310 (1784).-BEsser in Bull. Soc. Imp. Nat. Moseou VIII, p. 48 (1835).-Takeda in Bot. Mag. Tokyo XXV, p. 22 (1911).-Matsu mura, Ind. Pl. Jap. II-2, p. 624 (1912).NakaI in Bot. Mag. Tokyo XXVI, p. 99 (1912) (f. typica NakaI).-PAMPANINI in Nuovo Giorn. Bot. Ital. n. s. XXXIV, p. 660 (1927).-Tatew., Veg. Apoi p. 114 (1928).-Nakai, Veg. Apoi p. 71 (1930).-Masamune, Fl. \& Geobot. Stud. Yakusima p. 449 (1934).

Nom. Jap. Otoko-yomogi.

Hab. at the foot of Mt. Apoi, etc. Fl. Sep.

**;. resedifolia T TKKDA, l.c. p. 22 (1911).-NAKAI, l.c. (1912).

Syn. Artemisia japonica var. japonica Maxim. f. typica NaKaI subf. resedifolia Pampanini, l.c. p. 664 (1927).

Nom. Jap. Hosoba-no-otokoyomogi (Matsumura 1890).

Hab. at the foot of Mt. Apoi. Fl. Sep.

Dist. sp. Formosa, Liukiu, Kyushu, Shikoku, Honshu, Yezo Korea, China, Manchuria, Malaysia and Siberia.

$571^{(9)}$ *Artemisia Keiskeana Miques in Ann. Mus. Bot. Lugd.-Bat. II, p. 176 (1866).-Matsumura, Ind. Pl. Jap. II-2, p. 624 (1912).-NAKaI in Bot. Mag. Tokyo XXVI, p. 101 (1912) (f. hirtella NAKaI).-PAMPanini in Nuovo Giorn. Bot. Ital. n. s. XXXIV, p. 670 (1927) ; XXVI, p. 385 (1929).Tatew., Veg. Apoi p. 114 (1928).-Nakai, Veg. Apoi p. 71 (1930).-KitamURA in Acta Phytotax. et Geobot. V, p. 89 (1936).

Nom. Jap. Inu-yonogi.

Hab. at the foot of Mt. Apoi, Shoya, ete. Fl. Sep.

Dist. Kyushu, Shikoku, Honshu, Yezo, Korea, Manchuria, Ussuri and Amur.

$572^{(10)}$ Artemisia Koidzumii NaKaI in Bot. Mag. Tokyo XXV, p. 56 (1911) ; XXVI, p. 100 (1912).-Pampanini in Nuovo Giorn. Bot. Ital. n.s. XXXVI, p. 482 (1930) (var. typica PAMP.)-KITAMURA in Acta Phytotax. et Geobot. V, p. $90 \& 98$ (1936).

Syn. Artemisia vulgaris var. latiloba (non Ledebour) Kordzumi, Pl. Sachal. Nakahara. p. 120 (1910).-Kuno, Rep. Veg. N. Saghal. p. 234 
(1924).

Artemisia vulgaris var. latifolia (non Besser) TAKkDA in Bot. Mag. Tokyo XXV, p. 23 (1911).

Artemisia gilvescens (non Miquel) Pampanini, l.e. p. 465 (1930) (cum var. arguta PAMP.).

Artemisia ursorum Hultén, Fl. Kamt. IV, p. 188, fig. 6; pl. 6, fig. g (1930).

Nom. Jap. Hiroha-urajiro-yomogi (MiчaBe et Mryaki 1915).

Hab. in grassy places at the coasts of C. Erimo. FI. Sep.

Dist. Yezo, Kuriles, Saghalien, Korea, Ochotsk and Kamtchatka.

573(11)*Artemisia littoricola Kitamura in Acta Phytotax. et Geobot. V, p. $87 \& 94$ (1936).

Syn. Artemisia desertorum (non SpRengel) TaKeda in Bot. Mag. Tokyo XXV, p. 21 (1911).-NAKaI in Bot. Mag. Tokyo XXVI, p. 99 (1912). -Kudo, Rep. Veg. N. Saghal. p. 233 (1924).-NaKaI, Veg. Apoi p. 71 (1930).

Artemisia japonica var. macrocephala Pamp. f. sachalinensis PampaNINI in Nuovo Giom. Bot. Ital. n.s. XXXIV, p. 668 (1927).

Nom. Jap. Hama-otokoyomogi (Miyabe et Miyake 1915).

Hab. on coasts of Mt. Apoi, C. Erimo, Shoya, ete. Fl. Sep.

Dist. N. Honshu, Yezo, S. Kuriles, Saghalien and Korea?

Leaves are very polymorphous as in $A$. japonica Tirunzerg. Some has cuneate-obovate eauline leaves which are only serrate in the upper part (f. cuncata), and some pinnately parted Jeaves with linear, about $2 \mathrm{~mm}$ wide and sometimes laciniate lobes (f. pinnata).

574(12)* Artemisia sacrorum Lederour in Mém. Acad. Sci. St.-Pétersb. V, p. 571 (1805) ; Fl. Altaica IV, p. 72 (1833).

*var. laciniaeformis NAKAI in Bot. Mag. Tokyo XXVI, p. $101 \& 103$ (1912).

Syn. Artemisia sacrorum (non Ledebour) Matsumura, Ind. Pl. Jap. II-2, p. 625 (1912) pro partc.

Artemisia sacrorum var. major PAM. 1 , japonica subf. laciniacformis

Pampanini in Nuovo Giorn. Bot. Ital. n.s. XXXIV, p. 696 (1927).

*f. platyphylla Pampanini, l.c. p. 695 (1927) ; XXXVI, p. 387 (1929) (ut var. major f. platyphylla).

Syn. Artemisia sacrorum (non Ledebour) Matsumura, l.e. (1912) pro parte Artemisia sacrorum var. latiloba (non Ledebour) Takeda in Bot. Mag. Tokyo XXV, p. 22 (1911).-NAKAI, 1.c. p. 101 \& 102 (1912).Kudo, Rep. Veg. N. Saghal. p. 235 (1924). 
Artemisia sacrorum var. intermedia f. viridis (non LEDEBour) NAKAI, l.c. (1912).-TATEw., Veg. Apoi p. 114 (1928).

Artemisia Messerschmidtiana $\alpha$. viridis (non Besser) NaKaI, Fl. Sylv. Korea. XIV, p. 129, t. 27 (1923); Veg. Apoi p. 71 (1930).

Artemisia sacrorum var. major Pampanini, l.c. p. 694 (1927); l.c. p. 387 (1929).-Ki'ramura in Acta Phytotax. et Geobot. V, p. 87 (1936).

Nom. Jap. Iwa-yomogi.

Hab. on grassy coastal slopes at the foot of Mt. Apoi, Shoya, etc. Fl. mid.-late Sep.

Dist. var. Yezo, S. Kuriles, Saghalien, Korea and Manchuria.

$575^{(13)}$ *Artemisia Stelleriana Besser in Nouv. Mém. Soc. Nat. Moscou III, p. 79, pl. 5 (1834).-Franchet in Bull. Soc. Philom. Paris Ser. 7, XII, p. 87 (1888).-Matsumura, Ind. Pl. Jap. II-2, p. 625 (1912).-NakaI in Bot. Mag. Tokyo XXVI, p. 100 \& 102 (1912) incl. var. sachalinensis.-KUDo, Fl. Param. p. 169 (1922).-Pampanini in Nuovo Giorn. Bot. Ital. n.s. XXXIV, p. 704 (1927).-NAKaI, Veg. Apoi p. 71 (1930).-Hultén, F'l. Kamt. IV, p. 185 (1930).-Komarov, Fl. Penin. Kamt. III, p. 154 (1930).-Komarov et Klor.Alisovs, Key Pl. Far East. Reg. USSR. II, p. 1036, t. 311, f. 1, 6 \& 7 (1932). -Kitamura in Acta Phytotax. et Geobot. V, p. 88 (1936).

Nom. Jap. Shiro-yomogi.

Hab. on eoasts at the foot of Mt. Apoi, C. Erimo, Shoya, etc. Fl. mid. Jul.-Aug.

Dist. N. Honshu, Yezo, Kuriles, Saghalien, N. Korea, Ussuri and Kamtchatka. Naturalized in N. Europe and N. America.

Aster L.

$576^{(1+)}$ **Aster ageratoides Turczaninow in Bull. Soc. Imp. Nat. Moscon VII, p. 154 (1837).-Krtamura in Journ. Jap. Bot. XII, p. 642 (1936). * subsp. ovatus KiTAMURA, l.e. p. 647 (1936).

Syn. Aster trinervius var. ovata Franchet et Savatier, Enum. Pl. Jap. I, p. $222(1875)$.

**: var. yezoensis KITAMURA et HARA, var. nov.

Involucri squamae apice acutae vel acutiusculae purpureae. Flores purpurei. Cetera ut in subsp. ovato.

Nom. Jap. Yezo-nokongiku (nov.).

Hab. at the foot of Mt. Apoi. Fl. Aug.

Typus. prov. Ishikari : Jôzankei (H. Hara-Aug. 20, 1931).

Dist. var. Honshu and Yezo.

577(15)*Aster dubius ONNo in Biblioth. Bot. Ht. 106, p. 21 (1932).-KITAMURA in Journ. Jap. Bot. XII, p. 727 (1936). 
Syn. Inula dubia Thunberg, Fl. Jap. p. 318 (1784).

Erigeron Thunbergii A. GRAY, Bot. Jap. p. 395 (1859).

Erigeron dubius (non Sprengel 1826) Makino in Bot. Mag. Tokyo XVIII, p. 18 (1904).

subsp. glabratus (A. GraX) Kitamura et Hara, comb. nov.

Syn. Aster consanguineus Ledelour, Fl. Ross. II, p. 473 (1845).-HuLtén, Fl. Kamt. IV, p. 156 (1930).-Komarov, Fl. Penin. Kamt. III, p. 123 (1930).-Kitamura, l.c. p. 727 (1936).

Erigeron Thunbergii var. glabratum A. GraY, l.c. (1859).

Erigeron dubius var. alpicola Makino in MIyoshI et M $\triangle$ KINo, Alp. Pl. Jap. I, no.117 (1906).-MATSumura, Ind. Pl. Jap. II-2, p. 646(1912).

Erigeron alpicolus Makino in Bot. Mag. Tokyo XXVIII, p. 339 (1914).

Erigeron Thunbergii var. alpicolum Kudo, Fl. Param. p. 164 (1922). Nom. Jap. Miyama-adzumagiku.

IIab. in grassy places near Shoya, C. Erimo, Mt. Ruchishi, Mt. Toyoni, Mt. Rakko, ete. Fl. Jun.--Jul.

Dist. subsp. Honshu, Yezo, Kuriles, Saghalien, Korea, Ochotsk, Kamtchatka and E. Siberia.

The subspecies differs from $A$. dubius ONNo by having smaller tubeflowers, and shorter (ca. $3 \mathrm{~mm}$ long), whitish pappi. The specimen from C. Erimo has whitish pappi attaining $5 \mathrm{~mm}$ long, and is an intermediate form between the type and the subspecies.

*var. angustifolius (TATEWAKI) HARA, comb. nov.

Syn. Erigeron glabratus var. angustifolius TAtewaKI, Veg. Apoi p. 113 \& 131 (1928).

Erigeron glabratus (non Hoppe et Hornschuch) NakaI, Veg. Apoi p. $72(1930)$.

Erigeron Tsuneoi Tatewak. in Hokkaido Ringyô-kaihô 33, p. 594 (1935) cum fig.

Aster Tsuneoi Miyabe et Tatewaki in Trans. Sapporo Nat. Hist. Soc. XIV, p. 265, f. 15 (1936).

Aster consanguineus var. angustifolius HARA, mss.

Nom. Jap. Apoi-adzumagiku (TATEWaKi 1928).

Hab. in the upper \& middle part of Mt. Apoi and Mt. Horoman. Fl. late May-Jul.

Flowers are generally white, but sometimes, especially in the later season, purple or light blue. A glabrescent form with only sparsely shortpilose stems and involucral bracts also occurs (f. glabra HARA).

( $T$ o be continued). 